\title{
An Improved Sequence-based Prediction Protocol for Protein-Protein Interactions Using Amino Acids Substitution Matrix and Rotation Forest Ensemble Classifiers
}

\author{
Zhu-Hong You ${ }^{1}, \mathrm{Xiao} \mathrm{Li}^{1, *}$, Keith CC Chan ${ }^{2, *}$ \\ ${ }^{1}$ Technical Institute of Physics and Chemistry, Chinese Academy of Science \\ Urumqi 830011, China \\ ${ }^{2}$ Department of Computing, Hong Kong Polytechnic University \\ Hong Kong, P.R. China Xinjiang \\ *Correspondence: xiaoli@ms.xjb.ac.cn; keith.chan@polyu.edu.hk
}

\begin{abstract}
Protein-protein Interactions (PPIs) play important roles in a wide variety of cellular processes, including metabolic cycles, DNA transcription and replication, and signaling cascades. High-throughput biological experiments for identifying PPIs are beginning to provide valuable information about the complexity of PPI networks, but are expensive, cumbersome, and extremely timeconsuming. Hence, there is a need for accurate and robust computational methods for predicting PPIs. In this article, a sequence-based approach is proposed by combining a novel amino acid substitution matrix feature representation and Rotation Forest (RF) classifier. Given the protein sequences as input, the proposed method predicts whether or not the pair of proteins interacts. When performed on the PPI data of Saccharomyces cerevisiae, the proposed method achieved $93.74 \%$ prediction accuracy with $90.05 \%$ sensitivity at the precision of $97.08 \%$. Extensive experiments are performed to compare our method with the existing sequence-based method. Experimental results demonstrate that PPIs can be reliably predicted using only sequence-derived information. Achieved results show that the proposed approach offers an inexpensive method for computational construction of PPI networks, so it can be a useful supplementary tool for future proteomics studies.
\end{abstract}

Keywords: protein-protein interaction; substitution matrix; rotation forest; protein sequence; ensemble classifier;

\section{Introduction}

Protein-protein interactions (PPIs) play key roles in many cellular functions such as DNA transcription, metabolic cycles, and signaling cascades [1]. Until recently, several high-throughput experimental screens like Yeast two-hybrid screen (Y2H) [2], 
mass spectrometry [3], protein chip technology [4], and Tandem Affinity Purification tagging (TAP) [5] have been used for identifying PPIs for different species. Consequently, a large amount of PPI data for different organisms has been uncovered and recorded in public databases [6-9]. However, the biological experiments are not without shortcomings. Not only are they time-consuming and labor intensive, they are also extremely expensive. Another main disadvantage is that they yield relatively high rates of false-positive.

Hence, a reliable identification of PPIs with effective computational approach is of great significance. To date, a wide range of attempts have been made to develop computational methods for PPI identification. Broadly, these methods can be divided into several general categories: approaches based on evolutionary relationships, protein structure, protein domain, genomic information, and protein primary sequence[10]. Generally, the prediction accuracy of the first four is higher, but the deficiency of these approaches appears when they are exposed to problems without prior knowledge about proteins. Theoretically, the amino acids sequence of proteins contains all the necessary information to predict PPIs [11-13]. In addition, the advent of a complete genome sequence for many organisms has generated an enormous amount of protein sequence data for computational biologists [14-19]. Until now, a number of approaches for predicting PPIs directly from protein sequences are developed, and these works demonstrated that the information from protein sequences alone might suffice to predict PPI. There are two major challenges in the task of computational PPIs prediction from primary protein sequence, which are: a) how to effectively represent an amino acids sequence as a feature vector, and b) how to design a powerful computational model to accurately and fast predict the desired class (interacting or non-interacting)[20, 21].

For the first issue, the major challenge is how to supply a classifier with the features containing the interaction information to distinguish the interacting and noninteracting protein pairs. Currently, many protein sequence descriptors and features were adopted to represent amino acid sequence of a protein. Shen et al. developed the conjoint triad descriptor, which employs the frequency of three continuous amino acids to encoded protein sequences. When performed to predicting human PPIs, it achieves a high accuracy of $83.93 \%$ [22]. Guo et al. have developed a sequence-based method which yields a high prediction accuracy of $87.36 \%$, when applied to predicting the PPIs of $S$. cerevisiae. This method considers the interactions between residues a certain distance apart in protein sequence[23]. You et al. presented an ensemble model to predict PPIs using the information of protein sequence. When applied on the yeast dataset, it achieves $87.00 \%$ prediction accuracy with $86.15 \%$ sensitivity. Xia et al. also proposed a novel method for PPIs prediction by using a set of distinctive descriptors from protein sequences [24]. Although existing methods for PPIs prediction have been applied successfully, they still have some limitations and disadvantages [25-27]. For example, the sequence-order information of protein sequences is not fully considered. In this study, we aim to propose a novel protein feature representation method via the approach of amino acid substitution matrix and low rand approximation transformation. 
For the second issue, various machine learning methods such as Support Vector Machine (SVM), neural networks, decision tree classifier, Naïve Bayes, and ensemble classifier have been employed to construct the prediction model[28, 29]. Jansen et al. constructed a Naïve Bayes classifier to predict the interactions of proteins by integrating multiple features. [30]. You et al. propose a method for PPI prediction using Extreme Learning Machine model combined with a local protein sequence representation [26]. Bock et al. used a SVM model to classify the protein pairs using protein primary structure and associated physicochemical properties [31]. Other classification methods used in the prediction of PPIs are the k-Nearest Neighbor $(\mathrm{kNN})$, Logistic Regression (LR), Extreme Learning Machine (ELM), and Decision Trees (DT)[32, 33]. Among them, it has been shown that Random Forest model (RFD) consistently ranks as a top classifier, with SVM being in second place [34-39].

Rotation Forest is a recently proposed ensemble classifier, which is based on the idea of Random Forest. It was found to be more accurate than Random Forest classifier across a number of benchmark data sets [40]. In this article, we report a novel method for predicting protein-protein interactions using the rotation forest (RF) algorithm in conjunction with information of protein sequences. First, we transform each protein sequence into a substitution matrix, in which protein mutation information are contained. Then, a novel method termed as low-rank approximation (LRA) is introduced to find its approximate matrix, from which the row vector is extracted to numerically characterize each protein sequence. Finally, to improve the overall accuracy and robust for PPI prediction, rotation forest predictor is designed to carry out the PPI prediction. The proposed method was tested upon two PPI datasets. The experimental results demonstrate that the proposed approach is indeed feasible and effectual. Consequently, it is a new promising and powerful tool for large-scale PPI prediction.

\section{$2 \quad$ Materials and Methodology}

In this section, we describe the proposed approach for predicting protein interactions from protein sequences. Our method for predicting PPIs depends on three steps: (1) Map a protein sequence into a numerical matrix by using the amino acids substitution matrix feature representation; (2) The mapped matrix is analysed using Low-Rank Approximation technique to find its approximate matrix, from which a fixed length row vector is extracted to numerically characterize each protein sequence; (3) A Rotation Forest predictor is performed using the fixed length feature vector to predict the PPIs.

\subsection{Data Source}

In this study, three large, real public PPIs data sets are employed to evaluate the performance of the proposed method. The first dataset of physical protein interactions is collected from Saccharomyces cerevisiae core subset of database of interacting proteins (DIP), which is also used in the study of Guo et al. [41]. After the redundant protein pairs which contain a protein with fewer than 50 residues or have $\geq 40 \%$ sequence identity were remove, the remaining 5594 protein pairs comprise the final 
positive dataset. The 5594 non-interacting protein pairs were generated from pairs of proteins whose sub-cellular localizations are different. The whole dataset consists of 11188 protein pairs, where half are from the positive dataset and half are from the negative dataset. The second dataset is composed of 2,916 Helicobacter pylori protein pairs (1,458 non-interacting pair and 1,458 interacting pairs) as described by Martin et al. Other five species-specific PPI dataset including E.coli, C.elegans, H.sapiens, M.musculus, and H.pylori are employed in our experiment to verify the effectiveness of the proposed method. All the protein sequences in the seven datasets are extracted from database of SWISS-PROT. The dataset used in this study can be accessed at https://sites.google.com/site/zhuhongyou/data-sharing.

\subsection{Feature Vector Extraction}

\section{Substitution matrix for protein sequence}

To develop a powerful predictor for PPIs prediction, the key is how to design an effective feature representation method that can truly reflect the intrinsic information of protein sequences. The previous works show that the interacting proteins demonstrate similarity in the molecular phylogenetic tree because of the co-evolution through the interaction. Here we propose a novel feature representation method by incorporating the evolutionary information into protein sequence for PPIs prediction.

In the process of evolution, each amino acid is more or less likely to mutate into various other amino acids. For example, a hydrophilic residue such as arginine is more likely to be replaced by another hydrophilic residue such as glutamine. Formally, substitution matrix (SM) provides a description of the rate at which one residue in a sequence changes to other amino acid over time. The element in the $i$ th row and $j$ th column of a SM denotes the probability of the $i$ th amino acid being mutated to the $j$ th amino acid in the evolutional process. A 20 by 20 substitution matrix $M$ is shown in Figure 1 , and the element $M_{\mathrm{i}, \mathrm{j}}(i, j=1,2, \ldots, 20)$ represents the probability of amino acid $i$ mutating to amino acid $j$ during the evolution process. The matrix $M$ could be denoted as a 20 dimensional vector $M=\left(V_{1}, V_{2}, \ldots, V_{20}\right)$, where $V_{\mathrm{i}}=$ $\left(M_{1, \mathrm{i}}, M_{2, \mathrm{i}}, \ldots, M_{20, \mathrm{i}}\right)^{\mathrm{T}}$.

\begin{tabular}{|c|c|c|c|c|c|c|c|c|}
\hline & A & C & D & E & $F$ & G & H & $\longrightarrow$ \\
\hline A & 6 & -1 & -3 & -1 & -3 & 0 & -2 & \\
\hline c & -1 & 13 & -5 & -5 & -4 & -4 & -4 & \\
\hline D & -3 & -5 & 9 & 2 & -5 & -2 & -2 & \\
\hline$E$ & -1 & -5 & 2 & 7 & -5 & -3 & 0 & \\
\hline $\mathrm{F}$ & -3 & -4 & -5 & -5 & 9 & -5 & -2 & \\
\hline G & 0 & -4 & -2 & -3 & -5 & 8 & -3 & \\
\hline H & -2 & -4 & -2 & 0 & -2 & -3 & 11 & \\
\hline
\end{tabular}

Figure 1. An illustration of the amino acid substitution matrices. 
Given a protein sequence $S$ of length $L, \mathrm{~S}=\mathrm{s}_{1} \mathrm{~s}_{2} \cdots \mathrm{s}_{\mathrm{L}}$ over the 20-letter amino acids alphabet $\{A, C, D, E, F, G, H, I, K, L, M, N, P, Q, R, S, T, V, W, Y\}$. Let $\mathrm{s}_{\mathrm{i}}$ represents the ith amino acid of the protein sequence $S$ and could be replaced by a vector $V_{\text {si }}$ of the substitution matrix $M$. Then, we can easily obtain a 20 by $L$ matrix $\mathrm{D}=$ $\left(\mathrm{V}_{\mathrm{s} 1}, \mathrm{~V}_{\mathrm{s} 2}, \ldots, \mathrm{V}_{\mathrm{sL}}\right)$, where $L$ is the length of protein sequence and 20 dimensions mean a measure of residue conservation of 20 different standard amino acids.. In other words, the matrix representation for protein $S$ with $L$ amino acids can be represented by:

$$
D=\left(\mathrm{V}_{s 1}, \mathrm{~V}_{s 1}, \ldots, \mathrm{V}_{s L}\right)=\left[\begin{array}{cccc}
d_{1,1} & d_{1,2} & \cdots & d_{1, L} \\
d_{2,1} & d_{2,2} & \cdots & d_{2, L} \\
\vdots & \vdots & \vdots & \vdots \\
d_{20,1} & d_{20,2} & \cdots & d_{20, L}
\end{array}\right]
$$

where $d_{\mathrm{i}, \mathrm{j}}$ is the mutation probability, which reflects the probability of amino acid $i$ ( $i$ $=1,2, \ldots, 20)$ occurring at the sequence position $j(j=1,2, \ldots, L)$ in protein $S$ during evolutionary processes.

\section{Transformation from Substitution matrix to feature vector by Low-rank Ap-} proximation

Low-rank Approximation (LRA) is an important matrix analysis method, in which the cost function measures the fit between a given sparse matrix and an approximating matrix (the optimization variable), subject to a constraint that the approximating matrix has reduced rank. Here, using LRA upon the obtained protein feature matrix, we derive a matrix-based descriptor to represent the protein sequence. For a feature matrix $M$, which denotes a $20 * L$ matrix, the LRA of the data can be written as follows:

$$
\begin{aligned}
& \min _{\hat{M}}\|M-\hat{M}\|_{F} \\
& \text { Subject to: } \operatorname{rank}(\hat{M}) \leq r
\end{aligned}
$$

where $\|\bullet\|_{F}$ is the Frobenius norm. The above minimization problem has analytic solution in terms of the singular value decomposition (SVD) of the data matrix $M$.

Let $M=U \sum V^{T} \in R^{m \times n}$ be the SVD of $M$ and partition $U, \sum=: \operatorname{diag}\left(\sigma_{1}, \sigma_{2}, \sigma_{3}, \ldots, \sigma_{20}\right)$, and $N$ as follows:

$$
U=:\left[\begin{array}{ll}
U_{1} & U_{2}
\end{array}\right], \Sigma=:\left[\begin{array}{cc}
\Sigma_{1} & 0 \\
0 & \Sigma_{2}
\end{array}\right] \text {, and } V=:\left[\begin{array}{ll}
V_{1} & V_{2}
\end{array}\right]
$$

Where $\Sigma_{1}$ is a $r \times r$ matrix, $U_{1}$ is $m \times r$, and $V_{1}$ is $n \times r$. Then the rank- $r$ matrix is obtained as follows:

$$
\begin{gathered}
\hat{M}^{*}=U_{1} \sum_{1} V_{1}^{T} \\
\text { where }\left\|M-\hat{M}^{*}\right\|_{F}=\min _{\operatorname{rank}(\hat{M}) \leq r}\|M-\hat{M}\|_{F}=\sqrt{\sigma_{r+1}^{2}+\sigma_{r+2}^{2}+\ldots+\sigma_{m}^{2}}
\end{gathered}
$$

Then we compute the square root of the reduced matrix $\Sigma_{1}$ to obtain $\Sigma_{1}^{1 / 2}$ with dimensions $r$-by- $r$. Finally, we can get a $20 * r$ matrix $U_{1} \Sigma_{1}^{1 / 2}$, which contains the infor- 
mation of protein sequence order. It should be noticed that the feature matrix $M$ for different protein sequences sometime have different columns with each other, which shows that these protein primary sequences are non-equal length. However, the $U_{1} \Sigma_{1}^{1 / 2}$ for different protein sequences are $20 * r$ matrix.

We build a vector (row matrix) from the obtained matrix $U_{1} \Sigma_{1}^{1 / 2}$ by concatenating all rows, from 1 to 20 , of matrix $U_{1} \Sigma_{1}^{1 / 2}$. Therefore, the matrix-based protein descriptor consists of a total of $20 * r$ descriptor values, i.e., a $20 * r$ dimensional vector has been built to represent the protein sequence. Considering the trade-off between the overall prediction accuracy and computational complexity for extracting protein sequence descriptors, the optimal rank is $k=10$. Thus, we set $k$ to 10 in this study. A representation of an interaction pair is formed by concatenating the descriptors of two protein sequences in this protein pairs. Then, all descriptors from 20 rows were concatenated and a total 200 dimensional vector has been built to represent each protein sequence. Finally, the PPI pair is characterized by concatenating the two vector spaces of two individual proteins. Thus, a 400-dimentional vector has been constructed to represent each protein pair and used as a feature vector for input into rotation forest classifier.

\subsection{Rotation Forest Ensemble Classifier}

Rotation Forest is a relatively new ensemble learning algorithm, which is built from a set of decision tree. It was first proposed by Rodriguez et al and is based on bootstrap sampling [40, 42]. RF model provides diversity by using of Principal Component Analysis (PCA) which is used as axis rotation for subsets features. The framework of Rotation Forest can be described as follows: (1) the training dataset for each base classifier is generated by randomly splitting the original feature set into $K$ subsets; (2) PCA is applied to each of the subset. It should be noticed that all principal components are reserved in order to preserve all the information of the data; hence, features of base classifier is obtained with $K$ axis rotations. Actually, the diversity of RF algorithm is guaranteed by extracting features for each base classifier and accuracy is obtained by retaining all principal components and using the whole data set to train each base classifier [43]. (3) The testing instances are classified and the average confidence for each class across all base classifiers is calculated. The final class label is assigned to the one with the highest confidence value. The outputs of all trees are fused by the major vote rule. As RF classifier is more efficient and robust compared with many existing schemes, it has attracted many researchers' attention in recent years. The framework of rotation forest is described as follows, and more details can be referred to [40].

Consider a training sample set $S=\left\{\left(\mathrm{x}_{i}, \mathrm{y}_{i}\right)\right\}_{i=1}^{N}$ consisting of $N$ training samples, in which $\mathrm{X}_{i}$ denotes an input feature vector and $Y_{i}$ is the corresponding class label. Assuming that the feature set is randomly divided into $K$ subsets with the approximate size, there are $L$ decision trees in a rotation forest, denoted by, $D_{l}, \ldots, D_{L}$, respectively. $L$ and $K$ are the two parameters that should be determined in advance. The training dataset for an single classifier $D_{i}$ is preprocessed as following: 
(1) Divide $F$ into $K$ disjointed subsets randomly. As a result, each feature subset contains $M=n / K$ features. (2) Let $\mathrm{F}_{\mathrm{ij}}$ be the $j t h$ subset of features for training classifier $D_{\mathrm{i}}$ and $X_{\mathrm{ij}}$ be the dataset $X$ for the features in $\mathrm{F}_{\mathrm{ij}}$. For each subset, a non-empty subset of classes is selected from $X_{\mathrm{ij}}$ randomly. Then a bootstrap subset of objects is drawn with the size of 75 percent of the dataset to form a new training set, which is denoted by $X_{\mathrm{ij}}$ '. After that, the principal component analysis (PCA) technique is applied to $\mathrm{X}_{\mathrm{ij}}$ for generating the coefficients in a matrix $C_{\mathrm{ij}}$. Denoted by its coefficients. (3) Build a sparse rotation matrix $R_{i}$ with the obtained coefficients in matrix $C_{i j}$ as follows:

$$
R_{1}=\left[\begin{array}{cccc}
a_{i 1}^{(3)}, \ldots, a_{n 1}^{\left(M N_{1}\right)} & \{0\} & \ldots & \{0\} \\
\{0\} & a_{i 2}^{(0)} \ldots, a_{i 2}^{\left(M g_{2}\right)} & \ldots & \{0\} \\
\ldots & \ldots . & \ldots & \ldots \\
\{0\} & \{0\} & \ldots & a_{i K}^{(1)}, \ldots, a_{i K}^{\left(M N_{K}\right)}
\end{array}\right]
$$

The columns of $R_{\mathrm{i}}$ should be rearranged according to the original feature sequence, and assuming that the rearranged rotation matrix is denoted by $R_{\mathrm{i}}^{\mathrm{a}}$. Then the transformed training set for classifier $D_{\mathrm{i}}$ is $X R_{\mathrm{i}}^{\mathrm{a}}$. In this way, all classifiers will be trained in parallel.

In the classification phase, given a test sample $x$, let $d_{i j}\left(X R_{\mathrm{i}}^{\mathrm{a}}\right)$ be the probability produced by the classifier $D_{\mathrm{i}}$ to the hypothesis that $x$ belongs to class $i$. Then the confidence for a class can be calculated by the average combination method as follows:

$$
u_{j}(\mathrm{x})=\frac{1}{L} \sum_{i=1}^{L} d_{i j}\left(\mathrm{xR}_{i}^{a}\right), \mathrm{j}=1,2
$$

Then the test sample $x$ is easily assigned to the class with the largest confidence.

\section{Experiments and Results}

\subsection{Experimental setup}

In our experiments, the proposed sequence-based PPI predictor is implemented using MATLAB platform. All the simulations were carried out on a computer with Core 2 Duo CPU@3.1 GHz, 2CPUs and 12GB RAM, and Windows operating system.

\subsection{Evaluation measures}

To measure the performance of the proposed method, we adopted 5-fold cross validation and several parameters, the overall prediction accuracy (ACC), sensitivity (SN), specificity (SP), positive predictive value (PPV), negative predictive value (NPV), Fscore, and Matthews correlation coefficient (MCC). They are defined as follows:

$$
\begin{gathered}
A C C=\frac{T P+T N}{T P+F P+T N+F N} \\
S N=\frac{T P}{T P+F N} \\
S P=\frac{T N}{T N+F P}
\end{gathered}
$$




$$
\begin{gathered}
P P V=\frac{T P}{T P+F P} \\
N P V=\frac{T N}{T N+F N} \\
F 1=2 \times \frac{S N \times P P V}{S N+P P V} \\
M C C=\frac{T P \times T N-F P \times F N}{\sqrt{(T P+F N) \times(T N+F P) \times(T P+F P) \times(T N+F N)}}
\end{gathered}
$$

where true positive (TP) is the number of true PPIs that are predicted correctly; false negative (FN) is the number of true PPIs that are predicted to be non-interacting pairs; false positive (FP) is the number of true non-interacting pairs that are predicted to be PPIs, and true negative (TN) is the number of true non-interacting pairs that are predicted correctly. MCC denotes Mathew's correlation coefficient.

\subsection{Experimental results}

\section{Selecting the optimal $K$ and $L$}

In Rotation Forest model, the parameter $L$ represents the number of decision trees and $K$ denotes the number of features subsets. To select appropriate number of decision trees for RF model used in this study, we evaluate the proposed method with different ensemble size $L$ when the number of feature subsets $K$ is fixed to 10 . The evaluation performance is measured with 5-fold cross validation on yeast dataset. The experiment results are shown in Fig. 2. As can be seen, four curves show significant upward tendency when the number of decision trees increases to 21 . Then, as the number of decision trees increases, the plots flatten out gradually. More specifically, when the value of $L$ increases to 21 , four curves corresponding to overall accuracy, sensitivity, PPV, and MCC achieve their peaks, respectively. This suggests that the Rotation Forest classifier performs best when the number of decision trees is set to 21 .

Then, in order to investigate the impacts of parameter $K$, we evaluate the prediction performance using different $K$ when ensemble size $L$ is fixed to 21. Prediction results are shown in Fig. 3. As seen from the curves, with the increment of $K$, the classification results including overall accuracy, sensitivity, PPV, and MCC are significantly improved. Considering the tradeoffs between accuracy and computational complexity, the number of feature subsets is set to 10 . 


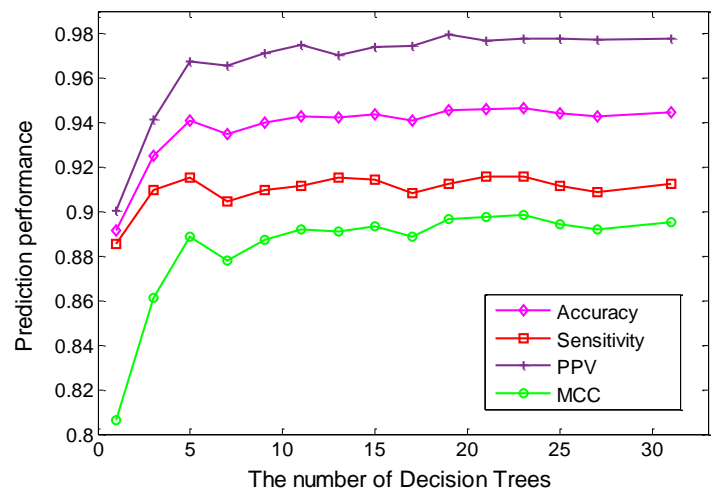

Figure 2. Selection of the optimal ensemble size of the Rotation Forest classifier.

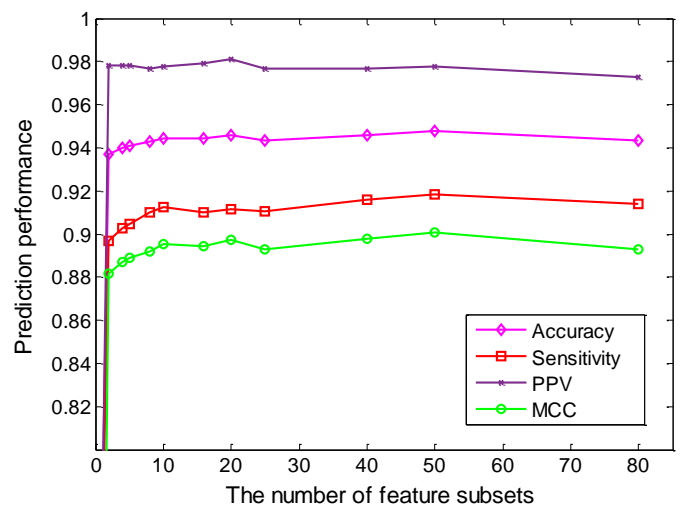

Figure 3. Selection of the optimal feature subsets number of the Rotation Forest classifier.

\section{Prediction performance of proposed model}

We evaluated the performance of the proposed model using the yeast PPIs data set as investigated in Guo et al. [41]. To guarantee that the experimental results are valid and can be generalized for making predictions regarding new data, the dataset is randomly partitioned into training and independent testing sets via a 5-fold cross validation. Each of the five subsets acts as an independent holdout testing dataset for the model trained with the rest of four subsets. Thus five models were generated for the five sets of data. The advantages of cross validation are that the impact of data dependency is minimized and the reliability of the results can be improved.

Table 1. Comparison of the prediction performance by the proposed method and some state-ofthe-art works on the yeast dataset. Here, N/A means not available.

\begin{tabular}{l|llllll}
\hline Model & Test set & $\mathrm{SN}(\%)$ & $\mathrm{PE}(\%)$ & $\mathrm{ACC}(\%)$ & $\mathrm{MCC}(\%)$ & $\mathrm{AUC}(\%)$ \\
\hline
\end{tabular}




\begin{tabular}{l|llllll} 
Our method & Average & $\mathbf{9 1 . 2 2} \pm \mathbf{1 . 6}$ & $\mathbf{9 7 . 1 0} \pm \mathbf{2 . 1}$ & $\mathbf{9 4 . 1 4} \pm \mathbf{1 . 8}$ & $\mathbf{8 8 . 9 6} \pm \mathbf{2 . 6}$ & $\mathbf{9 4 . 2 0} \pm \mathbf{1 . 7}$ \\
\hline \multirow{2}{*}{ Guos' work } & ACC & $89.93 \pm 3.68$ & $88.87 \pm 6.16$ & $89.33 \pm 2.67$ & N/A & N/A \\
& AC & $87.30 \pm 4.68$ & $87.82 \pm 4.33$ & $87.36 \pm 1.38$ & N/A & N/A \\
\hline $\begin{array}{l}\text { Zhous' } \\
\text { work }\end{array}$ & SVM+LD & $87.37 \pm 0.22$ & $89.50 \pm 0.60$ & $88.56 \pm 0.33$ & $77.15 \pm 0.68$ & $95.07 \pm 0.39$ \\
\hline \multirow{3}{*}{$\begin{array}{l}\text { Yangs' } \\
\text { work }\end{array}$} & Cod1 & $75.81 \pm 1.20$ & $74.75 \pm 1.23$ & $75.08 \pm 1.13$ & N/A & N/A \\
& Cod2 & $76.77 \pm 0.69$ & $82.17 \pm 1.35$ & $80.04 \pm 1.06$ & N/A & N/A \\
& Cod3 & $78.14 \pm 0.90$ & $81.86 \pm 0.99$ & $80.41 \pm 0.47$ & N/A & N/A \\
& Cod4 & $81.03 \pm 1.74$ & $90.24 \pm 1.34$ & $86.15 \pm 1.17$ & N/A & N/A \\
\hline
\end{tabular}

The prediction performance of rotation forest predictor with substitution representation of protein sequence across five runs are shown in Table 1, compared with several published results for the same yeast PPIs dataset. It can be observed from Table 1 that high prediction accuracy of $94.14 \%$ is obtained for the proposed model. To better investigate the prediction ability of our model, we also calculated the values of Sensitivity, Precision, MCC, and AUC. From Table 1, we can see that our model gives good prediction performance with an average Sens. value of $91.22 \%$, Prec. value of $97.10 \%$, MCC value of $88.96 \%$, and AUC value of $94.20 \%$. Further, it can also be seen in the Table 1 that the standard deviation of sensitivity, precision, accuracy, MCC and AUC are as low as 1.6\%, 2.1\%, 1.8\%, 2.6\% and 1.7\%, respectively.

We further compared our method with Guo et al.[41], Zhou et al.[44] and Yang et al.[45], where the SVM, SVM and KNN was performed with the conventional Auto Covariance, Local Descriptor, and Local Descriptor representation as the input feature vectors, respectively. From Table 1, we can see that the performance of all of these methods with different machine learning model and sequence based feature representation are lower than ours. To sum up, we can readily conclude that the proposed approach generally outperforms the previous model with higher discrimination power for predicting PPIs based the information of protein sequences. Table 2 plots the performance from the pruned and unpruned tree on the yeast dataset (Here we set $K=20$, $L=31$ ). It can be seen that the overall accuracy of unpruned tree is a little bit better than pruned tree.

Therefore, we can see clearly that our model is a much more appropriate method for predicting new protein interactions compared with the other methods. Consequently, it makes us be more convinced that the proposed method can be very helpful in assisting the biologist to assist in the design and validation of experimental studies and for the prediction of interaction partners. 
Table 2. Comparison of the prediction performance between pruned decision tree and unpruned decision tree on the yeast dataset.

\begin{tabular}{l|ccccccc}
\hline Model & $\mathrm{SN}(\%)$ & $\mathrm{SP}(\%)$ & $\operatorname{PPV}(\%)$ & $\mathrm{NPV}(\%)$ & $\mathrm{F} 1(\%)$ & $\mathrm{ACC}(\%)$ & $\mathrm{MCC}(\%)$ \\
\hline Pruned Tree & 90.05 & 97.35 & 97.08 & 90.92 & 93.43 & 93.74 & 88.23 \\
& 91.05 & 97.68 & 97.51 & 91.62 & 94.17 & 94.37 & 89.35 \\
\hline
\end{tabular}

\section{Comparing the prediction performance with other methods}

In order to highlight the advantage of the proposed model, it was also evaluated on Helicobacter pylori dataset. [46]. This dataset gives a comparison of proposed method with other previous works including phylogenetic bootstrap[47], signature products[46], HKNN[48], ensemble of HKNN[49] and boosting[50]. The methods of phylogenetic bootstrap, signature products and HKNN are based on individual classifier system to infer PPIs, while the methods of HKNN and boosting belong to ensemble-based classifiers. The average prediction results of 10-fold cross-validation over 6 different methods are shown in Table 3. From Table 3, we can see that the average prediction performance, i.e. sensitivity, PPV, accuracy and MCC achieved by proposed predictor, are $87.70 \%, 90.03 \%, 88.34 \%$ and $79.35 \%$, respectively. It demonstrates that our method outperforms all other individual classifier-based methods such as Phylogenetic bootstrap. We also observed that the proposed method clearly achieves a comparable performance with the other ensemble classifier systems (i.e. ensemble of HKNN and Boosting). All these results show that the proposed method not only achieves accurate performance, but also substantially improves precision in the prediction of PPIs.

Table 3. Performance comparison of different methods on the H.pylori dataset. Here, N/A means not available.

\begin{tabular}{l|cccc}
\hline \multicolumn{1}{c|}{ Methods } & SN (\%) & PPV (\%) & ACC (\%) & MCC (\%) \\
\hline Phylogenetic bootstrap & 69.8 & 80.2 & 75.8 & N/A \\
HKNN & 86 & 84 & 84 & N/A \\
Signature products & 79.9 & 85.7 & 83.4 & N/A \\
Ensemble of HKNN & 86.7 & 85 & 86.6 & N/A \\
Boosting & 80.37 & 81.69 & 79.52 & 70.64 \\
Proposed method & $\mathbf{8 7 . 7 0}$ & $\mathbf{9 0 . 0 3}$ & $\mathbf{8 8 . 3 4}$ & $\mathbf{7 9 . 3 5}$ \\
\hline
\end{tabular}




\section{Discussion and Conclusions}

This article proposes an accurate and robust computational method for predicting PPI based on only protein amino acids sequence. The proposed method combines the low rank approximation and rotation forest model for the first time. It yields high performance indicating that the LRA feature representation is very useful for the PPI prediction. It is well known that the mutation and distribution of amino acid possess more information than those of only composition. The main advantage of the proposed method is that it can extract more interaction information hidden in amino acid of protein sequence than traditional frequency-based methods can do. Furthermore, the application of rotation forest predictor ensures accurate and robust recognition of PPI. Experimental results well demonstrate that the proposed approach performs significantly well in distinguishing interacting and non-interacting protein pairs. Hence, it can be readily applied to meet the industrial needs.

Acknowledgments. This work is supported in part by the National Science Foundation of China, under Grants 61373086, 61572506, and in part by the Pioneer Hundred Talents Program of Chinese Academy of Sciences. The authors would like to thank all anonymous reviewers for their constructive advices.

\section{Reference:}

1. Zhang QC, Petrey D, Deng L, Qiang L, Shi Y, Thu CA, Bisikirska B, Lefebvre C, Accili D, Hunter T: Structure-based prediction of protein-protein interactions on a genome-wide scale. Nature 2012, 490(7421):556-560.

2. Krogan NJ, Cagney G, Yu HY, Zhong GQ, Guo XH, Ignatchenko A, Li J, Pu SY, Datta N, Tikuisis AP et al: Global landscape of protein complexes in the yeast Saccharomyces cerevisiae. Nature 2006, 440(7084):637-643.

3. Ho Y, Gruhler A, Heilbut A, Bader GD, Moore L, Adams SL, Millar A, Taylor P, Bennett K, Boutilier $\mathrm{K}$ et al: Systematic identification of protein complexes in Saccharomyces cerevisiae by mass spectrometry. Nature 2002, 415(6868):180-183.

4. Ito T, Chiba T, Ozawa R, Yoshida M, Hattori M, Sakaki Y: A comprehensive two-hybrid analysis to explore the yeast protein interactome. P Natl Acad Sci USA 2001, 98(8):45694574.

5. Gavin AC, Bosche M, Krause R, Grandi P, Marzioch M, Bauer A, Schultz J, Rick JM, Michon AM, Cruciat CM et al: Functional organization of the yeast proteome by systematic analysis of protein complexes. Nature 2002, 415(6868):141-147.

6. Chatr-aryamontri A, Breitkreutz B-J, Heinicke S, Boucher L, Winter A, Stark C, Nixon J, Ramage L, Kolas N, O'Donnell L: The BioGRID interaction database: 2013 update. Nucleic acids research 2013, 41(D1):D816-D823.

7. Orchard S, Ammari M, Aranda B, Breuza L, Briganti L, Broackes-Carter F, Campbell NH, Chavali G, Chen $\mathrm{C}$, del-Toro N: The MIntAct project-IntAct as a common curation platform for 11 molecular interaction databases. Nucleic acids research 2014, 42(D1):D358-D363. 
8. Veres DV, Gyurkó DM, Thaler B, Szalay KZ, Fazekas D, Korcsmáros T, Csermely P: ComPPI: a cellular compartment-specific database for protein-protein interaction network analysis. Nucleic acids research 2014:gku1007.

9. You ZH, Zhu L, Zheng CH, Yu HJ, Deng SP, Ji Z: Prediction of protein-protein interactions from amino acid sequences using a novel multi-scale continuous and discontinuous feature set. Bmc Bioinformatics 2014, 15 Suppl 15(Suppl 15):S9-S9.

10. Xia J-F, Han K, Huang D-S: Sequence-Based Prediction of Protein-Protein Interactions by Means of Rotation Forest and Autocorrelation Descriptor. Protein Peptide Lett 2010, 17(1):137-145.

11. Xia JF, Zhao XM, Huang DS: Predicting protein-protein interactions from protein sequences using meta predictor. Amino Acids 2010, 39(5):1595-1599.

12. De-Shuang H, Lei Z, Kyungsook H, Suping D, Kai Y, Hongbo Z: Prediction of proteinprotein interactions based on protein-protein correlation using least squares regression. Current Protein \& Peptide Science 2014, 15(6):553-560(558).

13. Yu HJ, Huang DS: Graphical Representation for DNA Sequences via Joint Diagonalization of Matrix Pencil. Biomedical \& Health Informatics IEEE Journal of 2013, 17(3):503-511.

14. You Z-H, Lei Y-K, Gui J, Huang D-S, Zhou X: Using manifold embedding for assessing and predicting protein interactions from high-throughput experimental data. Bioinformatics 2010, 26(21):2744-2751.

15. Bing W, Peng C, Huang DS, Li JJ, Lok TM, Lyu MR: Predicting protein interaction sites from residue spatial sequence profile and evolution rate. Febs Letters 2006, 580(2):380384.

16. Zhu L, Deng SP, Huang DS: A Two-Stage Geometric Method for Pruning Unreliable Links in Protein-Protein Networks. Nanobioscience IEEE Transactions on 2015, 14:528534.

17. Deng SP, Zhu L, Huang DS: Mining the bladder cancer-associated genes by an integrated strategy for the construction and analysis of differential co-expression networks. BMC Genomics 2015, 16 Suppl 3.

18. Yu HJ, Huang DS: Normalized feature vectors: A Novel Alignment-Free Sequence Comparison Method Based on Numbers of Adjacent Amino Acids. IEEE/ACM Transactions on Computational Biology \& Bioinformatics 2013, 10(2):457-467.

19. Zhu L, You Z-H, Huang D-S, Wang B: t-LSE: A Novel Robust Geometric Approach for Modeling Protein-Protein Interaction Networks. PLoS One 2013, 8(4):e58368.

20. Lei YK, You ZH, Zhen J, Lin Z, Huang DS: Assessing and predicting protein interactions by combining manifold embedding with multiple information integration. Bmc Bioinformatics 2012, 13(9):S3.

21. Zhu L, You ZH, Huang DS: Increasing the reliability of protein-protein interaction networks via non-convex semantic embedding. Neurocomputing 2013, 121(18):99-107.

22. Pan X-Y, Zhang Y-N, Shen H-B: Large-Scale Prediction of Human Protein-Protein Interactions from Amino Acid Sequence Based on Latent Topic Features. Journal of Proteome Research 2010, 9(10):4992-5001. 
23. Guo $\mathrm{Y}, \mathrm{Yu} \mathrm{L}$, Wen $\mathrm{Z}$, Li M: Using support vector machine combined with auto covariance to predict proteinprotein interactions from protein sequences. Nucleic Acids Research 2008, 36(9):3025-3030.

24. Xia J-F, Zhao X-M, Huang D-S: Predicting protein-protein interactions from protein sequences using meta predictor. Amino Acids 2010, 39(5):1595-1599.

25. Luo X, You Z, Zhou M, Li S, Leung H, Xia Y, Zhu Q: A Highly Efficient Approach to Protein Interactome Mapping Based on Collaborative Filtering Framework. Scientific Reports 2015, 5:7702-7702.

26. You Z-H, Lei Y-K, Zhu L, Xia J, Wang B: Prediction of protein-protein interactions from amino acid sequences with ensemble extreme learning machines and principal component analysis. BMC bioinformatics 2013, 14(Suppl 8):S10.

27. You Z-H, Li S, Gao X, Luo X, Ji Z: Large-Scale Protein-Protein Interactions Detection by Integrating Big Biosensing Data with Computational Model. BioMed Research International 2014, 2014.

28. Wang B, Huang DS, Jiang C: A New Strategy for Protein Interface Identification Using Manifold Learning Method. IEEE Transactions on Nanobioscience 2014, 13(2):118-123.

29. You Z-H, Yin Z, Han K, Huang D-S, Zhou X: A semi-supervised learning approach to predict synthetic genetic interactions by combining functional and topological properties of functional gene network. Bmc Bioinformatics 2010, 11(1):343.

30. Jansen R, Yu H, Greenbaum D, Kluger Y, Krogan NJ, Chung S, Emili A, Snyder M, Greenblatt JF, Gerstein M: A Bayesian networks approach for predicting protein-protein interactions from genomic data. Science 2003, 302(5644):449-453.

31. Bock JR, Gough DA: Predicting protein-protein interactions from primary structure. Bioinformatics 2001, 17(5):455-460.

32. Bader JS, Chaudhuri A, Rothberg JM, Chant J: Gaining confidence in high-throughput protein interaction networks. Nature biotechnology 2004, 22(1):78-85.

33. Zhang LV, Wong SL, King OD, Roth FP: Predicting co-complexed protein pairs using genomic and proteomic data integration. BMC bioinformatics 2004, 5(1):38.

34. Qi Y, Bar - Joseph Z, Klein - Seetharaman J: Evaluation of different biological data and computational classification methods for use in protein interaction prediction. Proteins: Structure, Function, and Bioinformatics 2006, 63(3):490-500.

35. You ZH, Li S, Gao X, Luo X, Ji Z: Large-Scale Protein-Protein Interactions Detection by Integrating Big Biosensing Data with Computational Model. Biomed Research International 2014, 2014:598129-598129.

36. You Z-H, Yu J-Z, Zhu L, Li S, Wen Z-K: A MapReduce based Parallel SVM for LargeScale Predicting Protein-Protein Interactions. Neurocomputing 2014.

37. Lei YK, Zou JW, Dong T, You ZH, Yuan Y, Hu Y: Orthogonal locally discriminant spline embedding for plant leaf recognition ${ }^{3}$. Computer Vision \& Image Understanding 2014, 119(2):116-126.

38. Mao $\mathrm{R}, \mathrm{Xu} \mathrm{H}, \mathrm{Wu} \mathrm{W}, \mathrm{Li} \mathrm{J}$ : Overcoming the challenge of variety: big data abstraction, the next evolution of data management for AAL communication systems. IEEE Communications Magazine 2015, 53(1):42-47. 
39. Ji Z, Wang B, Deng SP, You Z: Predicting dynamic deformation of retaining structure by LSSVR-based time series method. Neurocomputing 2014, 137(15):165-172.

40. Rodriguez JJ, Kuncheva LI, Alonso CJ: Rotation forest: A new classifier ensemble method. Pattern Analysis and Machine Intelligence, IEEE Transactions on 2006, 28(10):16191630 .

41. Guo YZ, Yu LZ, Wen ZN, Li ML: Using support vector machine combined with auto covariance to predict proteinprotein interactions from protein sequences. Nucleic Acids Res 2008, 36(9):3025-3030.

42. Liu KH, Huang DS: Cancer classification using Rotation Forest. Computers in Biology \& Medicine 2008, 38(5):601-610.

43. Zhang C-X, Zhang J-S: RotBoost: A technique for combining Rotation Forest and AdaBoost. Pattern Recogn Lett 2008, 29(10):1524-1536.

44. Zhou YZ, Gao Y, Zheng YY: Prediction of Protein-Protein Interactions Using Local Description of Amino Acid Sequence. In: Advances in Computer Science and Education Applications, Pt Ii. Edited by Zhou M, Tan HH, vol. 202. Berlin: Springer-Verlag Berlin; 2011: 254-262.

45. Yang L, Xia JF, Gui J: Prediction of Protein-Protein Interactions from Protein Sequence Using Local Descriptors. Protein Peptide Lett 2010, 17(9):1085-1090.

46. Martin S, Roe D, Faulon JL: Predicting protein-protein interactions using signature products. Bioinformatics 2005, 21(2):218-226.

47. Bock JR, Gough DA: Whole-proteome interaction mining. Bioinformatics 2003, 19(1):125-134.

48. Nanni L: Hyperplanes for predicting protein-protein interactions. Neurocomputing 2005, 69(1-3):257-263.

49. Nanni L, Lumini A: An ensemble of K-local hyperplanes for predicting protein-protein interactions. Bioinformatics 2006, 22(10):1207-1210.

50. Shi M-G, Xia J-F, Li X-L, Huang D-S: Predicting protein-protein interactions from sequence using correlation coefficient and high-quality interaction dataset. Amino Acids 2010, 38(3):891-899. 